\title{
Herbaria Mundi: An image centric approach to manipulation of specimens from multiple collections
}

\author{
Roger Hyam $¥$ \\ ‡ Royal Botanic Garden Edinburgh, Edinburgh, United Kingdom
}

Corresponding author: Roger Hyam (rhyam@rbge.org.uk)

Received: 28 Sep 2020 | Published: 05 Oct 2020

Citation: Hyam R (2020) Herbaria Mundi: An image centric approach to manipulation of specimens from multiple collections. Biodiversity Information Science and Standards 4: e59055. https://doi.org/10.3897/biss.4.59055

\begin{abstract}
Addressing the challenges of the Climate Emergency and the Biodiversity Crisis requires us to understand how the world's vegetation is changing. This is not a trivial task,especially in highly diverse tropical areas.

Frequently, the only way to characterise vegetation is to make voucher specimens of the plants present and identify them later in the lab.

Fortunately there are extensive reference collections carefully curated in herbaria. Unfortunately specimens and expertise are dispersed over hundreds of different herbaria spread across many countries. It would be more efficient if scientists could see and manipulate specimens independently of where they are stored, especially as travel becomes more difficult due to both the need to reduce carbon emissions and virus spread.

Herbaria Mundi is an application demonstrating how how this can be achieved using CETA F Specimen IDs and IIIF (International Image Interoperability Framework). The application mimics the way a botanist might work in a physical herbarium by enabling the gathering of specimens into groups and side-by-side comparison of specimens, but differs in that one can search for and manipulate specimens in multiple herbaria as if they were stored in a single collection.
\end{abstract}


Voucher specimens are often not added to herbaria mainly because they do not add enough value to material already in the collections. Herbaria Mundi therefore includes the ability for researchers to upload specimen images to Zenodo and for them to appear in the user interface as if they were in an institutional herbarium.

This proof of concept application is being developed as part of Task 4.3 of the Synthesys+ project funded by the European Commission.

Two things need to occur to take this concept into production.

1. More herbaria need to adopt the use of CETAF specimen identifiers and the IIIF image API.

2. Botanists need to prioritise which features they would like developed first.

The poster will be a catalyst to discuss how these things can be achieved.

\section{Keywords}

herbarium specimens, imaging, voucher specimens, identification, Zenodo

\section{Presenting author}

Roger Hyam

\section{Presented at}

TDWG 2020

\section{Funding program}

Task 4.3 of the Synthesys + project funded by the European Commission

\section{Hosting institution}

Royal Botanic Garden Edinburgh 